\title{
Liposome-mediated Transfection of Intact Viral Particles Reveals that Plasma Membrane Penetration Determines Permissivity of Tissue Culture Cells to Rotavirus
}

\author{
Dorsey M. Bass, * Michael R. Baylor, “ Clark Chen, * Erich M. Mackow," Michel Bremont," and Harry B. Greenberg ${ }^{\ddagger 5 \|}$ \\ Departments of * Pediatrics, ${ }^{\ddagger}$ Microbiology, and ${ }^{\S}$ Medicine, Stanford University, Stanford, California 94305; and "Gastroenterology, \\ Palo Alto Veterans Administration Hospital, Palo Alto, California 94304
}

\begin{abstract}
Rotaviruses are an important cause of gastroenteritis in human infants. In vivo, rotavirus displays striking cell tropism with viral replication generally restricted to the villus tip enterocytes of the small intestine. We studied a panel of cell lines that vary significantly in their permissivity to rotavirus infection. $L$ cells and HEp2 cells were relatively resistant to rotavirus infection compared with permissive Ma104 cells and HT29 cells. RNA transcription among the cell lines was proportional to antigen synthesis making a translational or posttranslational block an unlikely source of observed differences in susceptibility. All of the cell lines bound and internalized radiolabeled virus equally well, as measured by escape from surface protease treatment. Analysis of the escape of cell bound virus from neutralizing monoclonal antibody revealed that rotavirus did not immediately enter an eclipse phase in nonpermissive cells, but was internalized in an infectious form for several hours, possibly sequestered within endocytic vacuoles. $L$ cells and HEp2 cells were as permissive as Ma104 and HT29 cells when rotavirus infection was mediated by transfection of single- or doubleshelled rotavirus particles with cationic liposomes (Lipofec$\operatorname{tin}^{\mathrm{TM}}$ ). Rotavirus cell tropism in tissue culture cells is determined by the ability of infecting virions to traverse the plasma membrane of the cells into the cytoplasmic compartment. ( $J$. Clin. Invest. 1992.90:2313-2320.) Key words: rotavirus • liposome $\bullet$ permissivity $\bullet$ membrane penetration
\end{abstract}

\section{Introduction}

Rotavirus, a major cause of infantile diarrhea, selectively infects differentiated enterocytes on the villi of the mammalian small intestine. Infection of other tissues does not generally occur during natural infection in immunocompetent hosts (1). Furthermore, rotavirus also exhibits host range and age restriction. Rotavirus isolates usually cause disease only in the species of origin, and very young mammals are much more susceptible to disease than mature animals. The mechanisms of the highly specific cell and tissue tropism and host range restrictions of rotavirus are probably multifactorial. Previous work has suggested that the expression of specific cellular receptors for rotavirus $(2,3)$ and extracellular proteolytic activation (4) or inactivation (5) may be important in these restrictions to rotavirus

Address correspondence to Dorsey M. Bass, M.D., Division of Pediatric Gastroenterology, Room s22, Stanford University Medical Center, Stanford, CA 94305-5119.

Received for publication 13 December 1991 and in revised form 27 July 1992

The Journal of Clinical Investigation, Inc.

Volume 90, December 1992, 2313-2320 replication in vivo. Individual rotavirus isolates also vary in their ability to infect various cells in vivo and in tissue culture. The ability of various rotavirus strains to grow in Ma104 cells (6) and cultured human hepatocytes (7) has been mapped to gene 4 , which encodes vp4, the viral hemagglutinin.

At the cellular level, susceptibility or resistance to viral infection may be determined at a variety of steps in the viral replicative cycle, such as cell surface binding, entry into the appropriate cellular compartment for replication, uncoating, transcription, translation, assembly, or release from the cell.

In the present study, we have used tissue culture cell lines of varying permissivity for rotavirus infection to further analyze rotavirus cellular tropism. Analysis of specific stages of the viral life cycle including binding, transcription, translation, and assembly of progeny virions failed to detect a defect in the replicative cycle in nonpermissive cells. To bypass the membrane penetration stage of rotavirus replication, we used cationic liposomes to transfect intact icosahedral single- $(\mathrm{SS})^{1}$ and double-shelled (DS) rotavirus particles into both permissive and nonpermissive cell lines. Using this technique, we found that nonpermissive cells could be as efficiently infected as permissive cells. The results of our studies suggest that penetration of the infecting virion through the cell plasma membrane into the cytoplasmic compartment is the limiting step in virus replication in two cell lines which are relatively resistant to rotavirus infection.

\section{Methods}

Cells and virus. Rhesus rotavirus (RRV) and the UK strain of rotavirus (8) were grown in Mal04 cells and purified by hydrofluorocarbon extraction and isopycnic centrifugation, as previously described (9). HT29 cells and L 929 cells (L cells) were obtained from American Type Culture Collection (Rockville, MD), while HEp 2 cells were the generous gift of Dr. Franco Ruggeri (Palo Alto Veterans Administration Medical Center, Palo Alto, CA). All cells were grown in Dulbecco's modified MEM (DMEM) supplemented with $10 \%$ FCS, L-glutamine, penicillin, and streptomycin in a $5 \% \mathrm{CO}_{2}$ incubator. For studies requiring trypsin-free virus, monolayers were infected with 5 peroxidase focus units ( $\mathrm{pfu} / \mathrm{cell}$ ) of trypsin-activated virus for $4 \mathrm{~h}$, the monolayers were washed, and $0.5 \mathrm{ml}$ of $1 \%$ aprotinin was added to prevent residual trypsin from cleaving vp4 on viral progeny.

Infection of cells with $R R V$. Cells were grown to confluence in $24-$ well plates and washed twice with serum-free medium before inoculation. Rotavirus was trypsin treated with $5 \mu \mathrm{g} / \mathrm{ml}$ trypsin (type IX: Sigma Chemical Co., St. Louis, MO) for $30 \mathrm{~min}$ at $37^{\circ}$ before inoculation. For studies of one-step viral yield, cells were inoculated with 5 $\mathrm{pfu} /$ cell of RRV and incubated for $1 \mathrm{~h}$ at $37^{\circ}$. The monolayers were

1. Abbreviations used in this paper: DMEM, Dulbecco's modified MEM; DS, double shelled; pfu, peroxidase focus units; RRV, rhesus rotavirus; $\mathrm{SS}$, single shelled. 
then washed twice and treated for 30 min with neutralizing $\mathrm{mAb} 159$ (ascites fluid at 1:500 dilution) to neutralize any residual input virus on the cell surface. The monolayers were washed again twice and incubated $16-18 \mathrm{~h}$ at $37^{\circ}$ with $5 \% \mathrm{CO}_{2}$. Monolayers were harvested by freeze-thawing twice and brief trichlorotrifluoroethane extraction. The aqueous extracts were then titered in 10-fold dilutions on 96-well plates of Ma104 cells by immunoperoxidase focus counting using hyperimmune guinea pig serum as previously described (10).

Rotavirus antigen syntheses. Monolayers of the cell lines were washed and inoculated with serial 10 -fold dilutions of trypsin-activated $\mathrm{RRV}$ for $1 \mathrm{~h}$, washed, and treated with $\mathrm{mAb} 159$ as noted above. After $13 \mathrm{~h}$, the monolayers were washed and fixed with cold methanol. Immunoperoxidase staining was used to enumerate infected cells. Results were expressed as foci/milliliter for each cell line.

Quantitation of RRV RNA in infected cells. The various cell lines were infected at $10 \mathrm{pfu} /$ cell with trypsin-activated RRV at $4^{\circ}$ for $1 \mathrm{~h}$, then washed twice and warmed to $37^{\circ}$. Cells were harvested by scraping at time (before warming), $30 \mathrm{~min}$, and $4 \mathrm{~h}$. Serial dilutions of the cells were bound to nitrocellulose membranes in a dot blot manifold (BioRad Laboratories, Richmond, CA), fixed with glutaraldehyde, and treated with proteinase $\mathrm{K}$ as previously described (11). The blots were probed with random-primed ${ }^{32} \mathrm{P}$-labeled probes derived from reverse transcription of RRV RNA (12).

Binding and internalization of $R R V$. Confluent monolayers in 24well dishes were washed twice and chilled to $4^{\circ}$. Purified, trypsin-activated, metabolically ${ }^{35}$ S-labeled RRV $\left(150,000 \mathrm{cpm}, \sim 10^{6} \mathrm{pfu}, 10\right.$ $\mathrm{pfu} /$ cell) was added, and the monolayers were incubated at $4^{\circ}$ with gentle rocking for $1-1.5 \mathrm{~h}$. For measurement of binding, the monolayers were washed three times with cold serum-free media, lysed with $2 \%$ SDS, and counted as previously described (13). Under these conditions $\sim 10 \%$ of the radiolabeled virus bound to the monolayer. Binding of infectious virions was determined by harvesting the monolayers after washing by freeze-thawing and trichlorotrifluorethane extraction. The resulting aqueous phase was titrated on Ma104 monolayers by immunoperoxidase focus counting. Internalization was determined by binding the ${ }^{35} \mathrm{~S}$-labeled virus as above, followed by warming to $37^{\circ}$ for $45 \mathrm{~min}$. The cells were then treated with $500 \mu \mathrm{g} / \mathrm{ml}$ proteinase $\mathrm{K}$ for 30 min at $4^{\circ}$, washed twice with ice-cold media containing $2 \mathrm{mM}$ PMSF and $10 \%$ FCS, lysed, and scintillation counted. Control experiments showed that $95 \%$ of virus bound at $4^{\circ}$ and not allowed to internalize by maintaining the monolayers at $4^{\circ}$ was digested under these conditions.

Infectivity of internalized $R R V$. Confluent monolayers were washed with serum-free DMEM and infected with trypsin-activated RRV ( $10 \mathrm{pfu} /$ cell $)$ at $4^{\circ}$ for $1 \mathrm{~h}$. The monolayers were washed again and warmed to $37^{\circ}$ for the indicated time intervals. After washing again, neutralizing $\mathrm{mAb} 159$ ascites fluid was added at a 1:500 dilution at $4^{\circ}$ for $30 \mathrm{~min}$. This treatment has been shown to neutralize all cell surface-bound RRV if the cells had not been warmed to permit viral entry $(10,13)$. The monolayers were washed three times more and the cell-associated infectious virus was recovered by freeze-thawing once and trichlorotrifluoroethane extraction. The resulting aqueous phase was titrated on Ma104 cells by peroxidase focus counting. Control wells (total virus bound) were harvested after binding without $\mathrm{mAb}$ treatment or warming.

Transfection of cells with DS and SS RRV particles. SS RRV was obtained from $\mathrm{CsCl}$ gradients, dialyzed against Tris saline buffer ( 10 $\mathrm{mM}$ Tris, $100 \mathrm{mM} \mathrm{NaCl}, 1.5 \mathrm{mM} \mathrm{CaCl}$ ) and treated for $30 \mathrm{~min}$ at $24^{\circ}$ with $10 \mathrm{mM}$ EDTA to ensure removal of any remaining outer capsid proteins. The SS RRV particles prepared under these conditions had no residual infectivity when inoculated onto Ma104 cells at concentrations up to $100 \mu \mathrm{g} / \mathrm{ml}$. Preliminary experiments (data not shown) lead to the development of the following protocol for the efficient lipofection of viral particles with minimal toxicity to the cells. Viral particles were serially diluted in serum-free DMEM beginning at $0.05 \mu \mathrm{g}$ protein $/ \mathrm{ml}$, (Lipofectin ${ }^{\mathrm{TM}}$; Bethesda Research Laboratories, Gaithersburg, MD) was added to $10 \mu \mathrm{g} / \mathrm{ml}$, and the mixture was vortexed briefly. Duration of the vortexing had little effect on the efficiency of the transfection (data not shown). Confluent monolayers were washed twice with serum-free DMEM. They were then transfected with 300 $\mu \mathrm{l} /$ well of the SS RRV-Lipofectin ${ }^{\mathrm{TM}}$ preparation. After $3-5 \mathrm{~h}$ at $37^{\circ}, 1$ $\mathrm{ml}$ of serum-free DMEM was added to each well. After $12 \mathrm{~h}$ of further incubation, the cells were either fixed and immunoperoxidase stained or harvested by freeze-thawing for titration of infectious progeny. Double-shell particles were transfected into cells by the same protocol with the exception that calcium-magnesium-free Joklik's modified MEM was used to minimize intracellular calcium fluxes during lipofection. The DS particles had been grown in the presence of trypsin $(0.5 \mu \mathrm{g} / \mathrm{ml})$ before purification. For mAb neutralization experiments, virions were preincubated with 1:500 dilutions of $\mathrm{mAb}$ ascites fluid for $30 \mathrm{~min}$ before the addition of Lipofectin ${ }^{\mathrm{TM}}$.

Effect of DEAE dextran on viral infectivity. Serum-free medium containing DEAE dextran (Sigma) at concentrations ranging from 25 to $400 \mu \mathrm{g} / \mathrm{ml}$ was added to washed cell monolayers for $30 \mathrm{~min}$ at $37^{\circ}$. Serial dilutions of purified SS or trypsin-activated DS RRV particles were added and viral attachment and entry were allowed to proceed for $2 \mathrm{~h}$ at $37^{\circ}$. The wells were washed twice with serum-free medium and incubated for an additional $8 \mathrm{~h}$ before fixation and quantitation of infected cells by immunoperoxidase staining.

\section{Results}

Susceptibility of cell lines to RRV infection. We examined several continuous cell lines for their ability to support replication of RRV. Ma104 cells are a simian renal epithelial cell line, which is the standard line used for propagation of rotaviruses. $\mathrm{L}$ cells are murine peritoneal fibroblasts that are commonly used in the propagation of mammalian reoviruses. HEp2 cells are human laryngeal epithelial cells commonly used to propagate enteroviruses and HT29 cells are colonic epithelial cells that under certain conditions may express certain phenotypic markers of small intestinal epithelial cells. A previous report has described their utility in propagating rotaviruses (14).

When we determined the yield of infections performed at

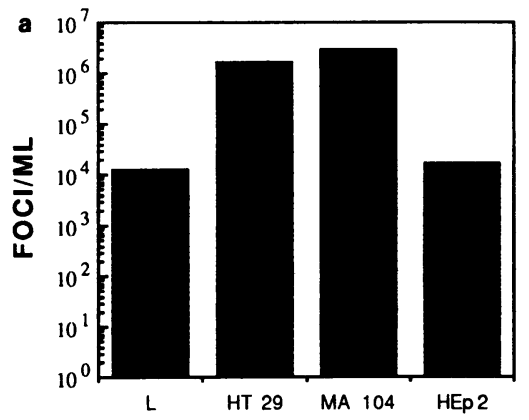

Figure 1. (a) Infectious yield of RRV infected cells. The indicated cell lines were infected with trypsin activated RRV at of $5 \mathrm{pfu} /$ cell for $1 \mathrm{~h}$ at $37^{\circ}$, then treated with neutralizing $\mathrm{mAb} 159$ to remove residual input virus, and harvested at $16 \mathrm{~h}$ as described in the text before titration of the yield on Ma104 cells. Data represents

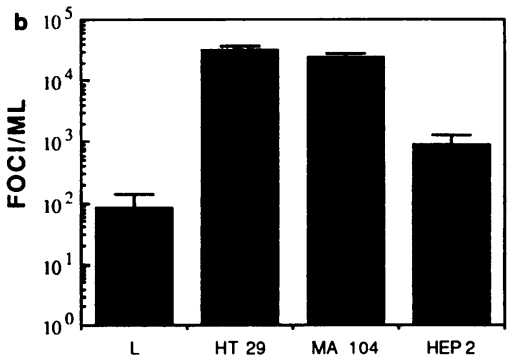
the average of replicate infection. The range was $\leq \pm 0.4$ log. (b) RRV antigen production in cell lines. The indicated cell lines were infected with serial dilutions of trypsin activated RRV for $1 \mathrm{~h}$, then treated with 1:500 dilution of neutralizing $\mathrm{mAb} 159$ to neutralize residual input virus and washed, as described in the text. The monolayers were methanol fixed at 12 $\mathrm{h}$ after infection before immunoperoxidase staining to identify infected cells. Results are the mean of triplicate infections with the SE indicated. 
high multiplicity of infection ( 5 pfu/cell), Ma104 and HT29 cells yielded between 100 and 300 times more virus than L cells and HEp2 cells (Figure $1 a$ ). To determine whether these differences in viral yield might be caused by late events in the viral replicative cycle, we determined the relative ability of the cell lines to synthesize viral antigen by immunoperoxidase staining-infected cells. Again, we found that the Ma104 and HT29 cells were between 100 - and 300 -fold more susceptible to RRV, as measured by the peroxidase focus assay (Figure $1 b$ ). Similar differences in the susceptibility of various cell lines to rotavirus (2-3 $\log _{10}$ ) have been previously reported (7). The total amount of viral antigen per cell, observed as judged by the intensity of the immunoperoxidase staining, appeared equivalent among the various cell lines but there were consistently fewer $L$ and HEp2 cells expressing viral antigen at a variety of viral input levels. Even at very high multiplicities of infection ( $10-50$ pfu / cell ) we were never able to infect more than 5-10\% of the HEp2 and L cells, as measured by immunoperoxidase labeling (data not shown).

The amount of infectious progeny virus per cell infected (antigen positive by immunoperoxidase staining) ranged between 146 foci/cell for L cells and 48 foci/cell for HT29 cells (Table I), indicating that each infected cell from the four cell lines produced a more or less equivalent amount of progeny virus. Similar patterns of antigen production among the four cell lines were observed using other rotaviral strains such as UK (bovine), Wa (human), and EB (murine) (data not shown). These results suggested that assembly and release of viral particles were not rate-limiting steps for the nonpermissive cell lines, since once a nonpermissive cell was infected, it produced comparable amount of viral antigen and infectious virus.

Transcription of RRV RNA. Having observed that the yield of infectious progeny virus was proportional to the antigen syntheses and the number of infected cells, we wished to determine whether a block in translation of RRV mRNA might account for the lower antigen yield in L cells and HEp2 cells. To answer this question, we determined the viral RNA transcript content of the cell lines at various times after infection. A semiquantitative Northern dot blot assay was used for this purpose. The results (Fig. 2) showed that RRV transcript levels were significantly higher ( $\geq 64$-fold) in the susceptible Ma104 and HT29 cells than in the relatively resistant $L$ and Hep 2 cell lines at $4 \mathrm{~h}$ after infection. The detected RNA was not part of the inocu-

Table I. Progeny RRV Virions Produced per Infected Cell*

\begin{tabular}{lr}
\hline Cell line & pfu/cell \\
\hline L cells & 146 \\
HT 29 & 49 \\
MA 104 & 125 \\
HEp 2 & 76
\end{tabular}

\footnotetext{
* The four cell lines in quadruplicate wells of 24-well plates were infected with 5 and $1 \mathrm{pfu} /$ cell RRV, as described in the text. After $1 \mathrm{~h}$ extracellular virus was neutralized with $\mathrm{mAb} 159$. The number of cells infected was determined in two wells by immunoperoxidase staining after $12 \mathrm{~h}$ of incubation. Progeny virus was determined in the two remaining wells by titration of cell lysates after $16 \mathrm{~h}$ of incubation on MA 104 cells by immunoperoxidase focus counting. The results are expressed as the viral yield (total pfu/well) divided by the total number of cells that were stained for viral antigen per well.
}

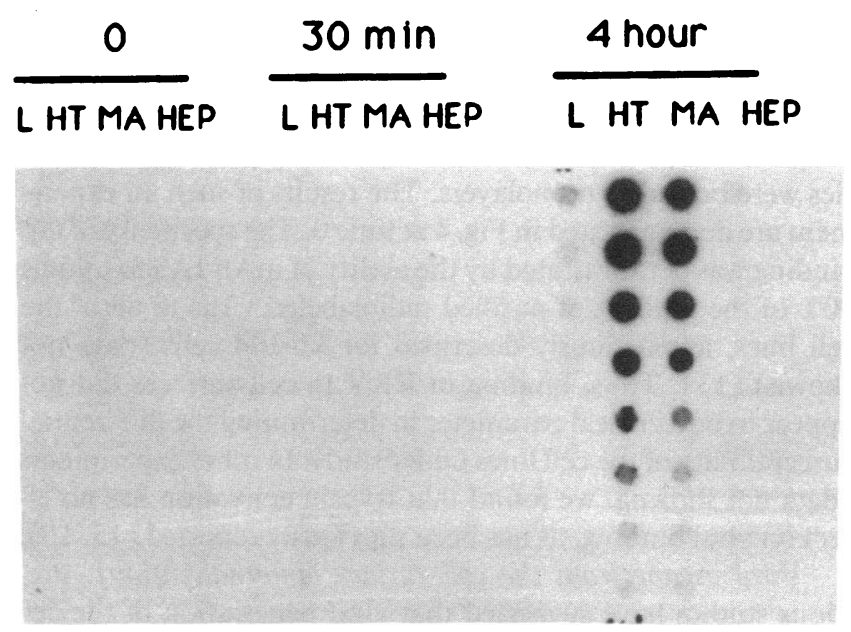

Figure 2. Northern dot blots of RRV infected cells. Indicated cell lines were infected with $10 \mathrm{pfu} \mathrm{RRV} /$ cell and then harvested at indicated times. Cells were bound to nitrocellulose as described in the text in serial twofold dilutions and probed with ${ }^{32} \mathrm{P}$-labeled random-primed RRV cDNA probe derived from total RRV RNA transcripts. The vertical axis represents serial twofold dilutions of the cells beginning at $10^{4}$ cells.

lum as demonstrated by the lack of reactivity of all of the cell lines at times 0 and $30 \mathrm{~min}$. The level of transcription roughly paralleled the level of infectious yield and the number of cells infected (Fig. 1, $a$ and $b$ ) in the permissive and nonpermissive cells. Thus, it appeared unlikely that inefficiency of translation per se impeded RRV replication in L and HEp cells, and it seemed likely that restriction occurred at a pretranslational step.

Binding of RRV to cells. Because previous work in our laboratory (2) and by others (3) has suggested that rotavirus tissue tropism and host range restriction might be related to the level of expression of specific viral receptors, we determined whether the permissive and nonpermissive cell lines differed in their capacity to bind purified, metabolically ${ }^{35} \mathrm{~S}$-labeled, infectious RRV. Results of a typical experiment, shown in Fig. 3, revealed

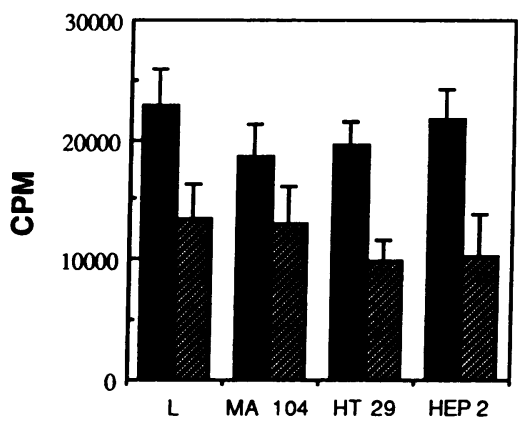

Figure 3. RRV cell binding and internalization. Binding was determined by incubating confluent monolayers with $150,000 \mathrm{cpm}$ of purified, trypsin-activated ${ }^{35}$ S-labeled $R R V$ $\left(10^{6} \mathrm{pfu}, 10 \mathrm{pfu} / \mathrm{cell}\right)$ at $4^{\circ}$ for $1 \mathrm{~h}$ before washing, solubalization, and scintillation counting. Binding of in-

fectious virus was determined by washing after $1 \mathrm{~h}$ of adsorption at $4^{\circ}$ followed by harvesting the cells and titration on MA 104 monolayers, as described in the text. Internalization of the labeled virus was determined by warming the monolayers for $45 \mathrm{~min}$ to $37^{\circ}$ after allowing virus to bind. Cells were then treated with $500 \mathrm{mg} / \mathrm{ml}$ proteinase $\mathrm{K}$ for $30 \mathrm{~min}$ at $4^{\circ}$ to remove surface-bound virus, washed, and solubalized for scintillation counting. Results represent the means of three experiments with SE indicated. $₫$, cpm bound; 0 , cpm internalized. 
that all of the cell lines had a more or less equal capacity to bind RRV measured by the amount of radiolabeled virus adsorbed to the monolayers at $4^{\circ}$. In several experiments, we demonstrated that equivalent amounts of potentially infectious particles were bound to monolayers. The results of such an experiment are demonstrated in Fig. 4 at time 0 . The specificity of the binding was demonstrated by the ability of mAb $1 \mathrm{~A} 9$ to inhibit $90 \%$ of the binding of purified radiolabeled virus to all of the cell lines, as previously described for Ma104 cells (data not shown) (13). Thus, binding of RRV to cell surfaces did not appear to be a critical parameter in determining the differential susceptibility of the cell lines under study. In other experiments (data not shown), we found that trypsin activation has no effect on viral binding, as has been previously reported $(15,16)$.

Viral escape from the cell surface (internalization). Previous studies have suggested that viral penetration of the cell surface membrane was a critical step in rotavirus replication that requires trypsin cleavage of the viral spike protein vp4 into $\mathrm{vp} 5^{*}$ and $\mathrm{vp} 8 *(10,17)$. In experiments designed to determine how much of the bound RRV entered the cells, prebound radiolabeled RRV was allowed to enter cells by warming them to $37^{\circ}$. After $45 \mathrm{~min}$, surface-bound virus was removed by proteinase $\mathrm{K}$ digestion. As seen in Fig. 3, 50-70\% of the bound virus was resistant to digestion in all of the cell lines under these conditions. If the monolayers were not warmed before proteinase $\mathrm{K}$ treatment, $<5 \%$ of the $\mathrm{cpm}$ remained cell associated. Trypsin activation of the radiolabeled virus before inoculation had no significant effect on the internalization of virus under the conditions used in this experiment (data not shown), as was previously reported (16). Similar results were obtained using trypsin-EDTA rather than proteinase $\mathrm{K}$ to remove virions from the cell surface (data not shown). Thus, a simple difference in internalization of the surface-bound virus, as measured by this assay, was not the determinant of the differential susceptibility observed.

Infectivity of internalized virus. Because no gross difference between the cell lines in either binding or internalization of the virus was observed, we decided to study the fate of internalized virus in the permissive and nonpermissive cell lines in more detail. Efforts to determine the biochemical characteristics of the internalized particles, either by SDS-PAGE or by characterizing the input particles by equilibrium centrifugation in $\mathrm{CsCl}$, as described by Ludert et al. (18), failed to reveal clear cut differences between susceptible and resistant cell lines (data not shown ). We therefore decided to examine the infectivity of internalized RRV. After allowing internalization at $37^{\circ}$ of prebound RRV ( $5 \mathrm{pfu} /$ cell) for various intervals, the monolayers were treated with anti-RRV vp7 mAb 159. This $\mathrm{mAb}$ has been shown previously to efficiently neutralize virus bound to the cell surface (13). The monolayers were then washed, harvested, and fluorocarbon extracted to release aggregated or membrane bound intracellular virus before titration of residual virus infectivity on Ma104 cells by immunoperoxidase staining.

Fig. 4 depicts the results of such an experiment. In the permissive cell lines (Ma104 and HT29), internalized virus rapidly lost infectivity over the first 120 min of infection. Presumably, much of this loss or eclipse was caused by viral uncoating and the initiation of transcription. In contrast, the RRV which had entered L cells and HEp2 cells retained a large degree of infectivity during the same interval. By $8 \mathrm{~h}$ (480 min), viral replication is evident in the permissive cells. These results sug-

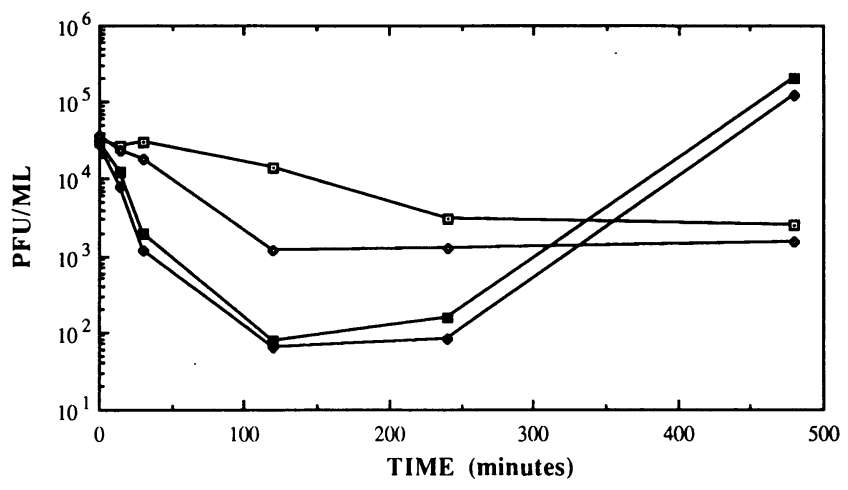

Figure 4. Residual infectivity of internalized RRV. The indicated cell lines were infected with $5 \mathrm{pfu} /$ cell trypsin-activated RRV, as noted in the text, at $4^{\circ}$. After various intervals at $37^{\circ}$, surface bound virus was neutralized with a 1:500 dilution of mAb 159 ascites at room temperature for $30 \mathrm{~min}$. Excess $\mathrm{mAb}$ was removed by washing twice and the cells were harvested and lysed as described in the text. The lysates were titered on MA 104 cells. Zero time lysates were prepared by freezing monolayers to which RRV had bound at $4^{\circ}$, but were not warmed or treated with $\mathrm{mAb}$. Data represent means of triplicate assays. The range of values obtained did not exceed $0.5 \log _{10} .-\odot-$, L cells; — -, HT29; —- -, MA 104; — -, HEP2.

gested that virus internalized by the two less susceptible cell lines either inefficiently escaped from endocytic vesicles and/ or was failing to uncoat efficiently to transcriptionally active but noninfectious single shell particles.

Lipofection of $S S$ and $D S R R V$ particles. After establishing that cell surface binding and escape from the cell surface were not the primary determinants of permissivity, we wished to examine whether viral penetration of the cell plasma membrane into the cell cytosol was restricted in the nonpermissive cells. To bypass this potential barrier to infection of nonpermissive cells, we used transfection via cationic liposomes ( $\mathrm{Li}-$ pofectin ${ }^{\mathrm{TM}}$ ) of single-shell particles into the cell lines. We reasoned that such a transfection strategy might be a way to nonspecifically target transcriptionally active SS RRV particles into the cytoplasm of both permissive and nonpermissive cells. We wished to learn if the nonpermissive cells could be rendered permissive if SS particles made their way into the cell cytoplasm. When analyzed by immunoperoxidase staining, we found that all of the cell lines were equally able to produce RRV antigen after lipofection of the noninfectious SS particles (Fig. $5 a$ ). If the transfected monolayers were analyzed for yield of infectious particles after a single cycle of replication (Fig. $5 b$ ), the titers of infectious progeny were quite similar. The calculated number of progeny virions per infected cell was similar among all four cell lines and comparable to that obtained during DS infection of the cell lines (Table II). In the absence of liposomes, single-shelled RRV particles produced no antigen or progeny virus. Similar results (infection of all cell lines equally) were obtained with purified single-shelled particles from the UK bovine strain rotavirus (data not shown).

Having determined that single-shelled particles could initiate a complete replicative cycle with equal efficiency in both permissive and nonpermissive cell lines if introduced into the cytoplasm, we wished to determine if the difference between the permissive and nonpermissive cells might be a defect in the cytoplasmic uncoating of double-shelled particles to the single shelled transcriptionally active form. It has been hypothesized 

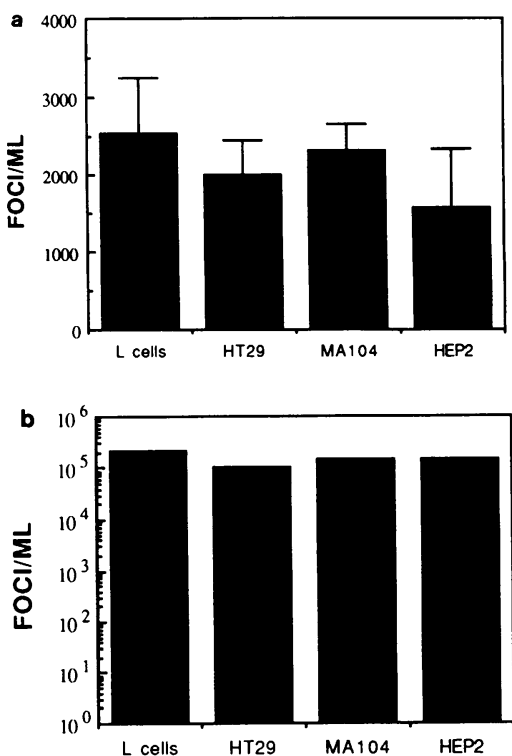

Figure 5. (a) Liposomemediated infection of cells by single-shelled RRV particles. Confluent plates of the indicated cell lines were washed with serum-free media and transfected with a mixture of 10 fold dilutions of CsCl-purified RRV SS particles (beginning at $0.05 \mu \mathrm{g} / \mathrm{ml}$ ) and Lipofectin ${ }^{\mathrm{TM}}(10 \mu \mathrm{g} / \mathrm{ml})$ for $4 \mathrm{~h}$, then refed with medium. Cells were fixed and immunoperoxidase stained at $12 \mathrm{~h}$ after infection. No peroxidase foci were observed in control wells inoculated with SS RRV alone.

Data represent the means of triplicate wells with SE indicated. (b) Infectious yield of cells transfected by liposomes with RRV SS particles. The indicated cell lines were inoculated with SS RRV particles $(5 \mu \mathrm{g} / \mathrm{ml})$ and liposomes $\left(10 \mu \mathrm{g} / \mathrm{ml} \mathrm{Lipofectin}^{\mathrm{TM}}\right)$; as noted in the text, the inoculum was removed and replaced with fresh medium after $5 \mathrm{~h}$. After $14 \mathrm{~h}$ the cells were lysed and titered on MA 104 cells. Data represent means of duplicate transfected wells where the range was $< \pm 0.5 \log$. No infectious progeny were detected in wells inoculated with SS RRV without Lipofectin ${ }^{\mathrm{TM}}$.

that rotavirus uncoating occurs as the virus reaches the low calcium environment of the cytosol (18). In vitro calcium chelation by EDTA or EGTA results in uncoating and activation of the viral transcriptase (19). We therefore repeated our transfection experiments with $\mathrm{CsCl}$ gradient purified double-shell particles. The results, shown in Fig. 6, indicated that cationic liposomes greatly enhanced the infectivity of double-shelled RRV in the less susceptible cell lines, such that these cells were essentially indistinguishable from permissive cells under these conditions. Control wells inoculated with the same doubleshelled RRV preparation at the same concentration but without liposomes showed the expected low efficiency of infection in L and HEp2 cells. Likewise the use of Lipofectin ${ }^{\mathrm{TM}}$ greatly

Table II. Progeny RRV Virions Produced per Cell Transfected with Single-shell $R R V^{*}$

\begin{tabular}{lc}
\hline Cell line & pfu/cell \\
\hline L cells & 129 \\
HT 29 & 78 \\
MA 104 & 101 \\
HEp 2 & 140
\end{tabular}

\footnotetext{
* Confluent monolayers were transfected with $5 \mu \mathrm{g} / \mathrm{ml} \mathrm{SS} \mathrm{RRV} \mathrm{par-}$ ticles via cationic liposomes (Lipofectin ${ }^{\mathrm{TM}}$ ), as described in the text. Duplicate sets of monolayers were either fixed and immunoperoxidase stained at $10 \mathrm{~h}$ for determination of the number of infected cells/well or harvested as cell lysates and titrated on MA 104 cells to determine the number of infectious progeny virions. RRV SS particles without lipofectin yielded no infected cells or progeny.
}

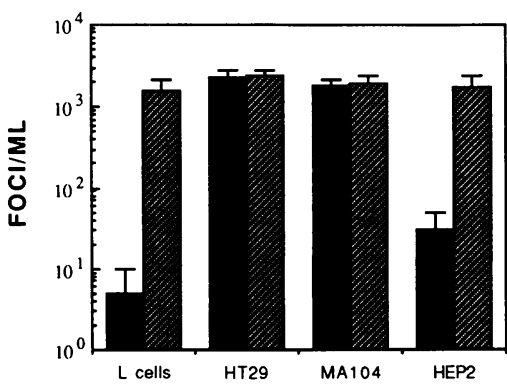

Figure 6. Liposome-mediated infection of cell lines by double-shelled RRV. Transfections using $\mathrm{CsCl}$ purified $\mathrm{RRV}$ DS particles $(0.1 \mathrm{pfu} /$ cell) and Lipofectin ${ }^{\mathrm{TM}}$ (DS L) or DS infections in the absence of Lipofectin $^{\mathrm{TM}}$ (DS C) were performed as described in the text. Infected cells

were quantitated by immunoperoxidase staining. Data are means from triplicate wells with SE indicated. $₫$, DS C; , DS L.

enhanced infection of $\mathrm{L}$ and HEp2 cells by purified doubleshelled UK virions (data not shown).

Because it was possible that the purified DS RRV preparations might contain some SS particles that might have been responsible for the liposome-mediated infection, we used neutralizing $\mathrm{mAb}$ to confirm that the observed Lipofectin ${ }^{\mathrm{TM}}$-mediated infection was caused by DS particles. $\mathrm{mAb} 159$ is a potent neutralizing antibody directed against the outer capsid protein vp7. When the DS particles were preincubated with mAb 159 before the Lipofectin ${ }^{\mathrm{TM}}$-mediated transfection, no infected cells were observed (Fig. 7). Nonneutralizing mAbs 129 (anti-vp7) and 255/60 (anti-vp6) had no effect on transfection of double-shelled particles (data not shown), while mAb 159 had no effect on transfection of single-shelled particles. Thus, the enhanced infectivity of the lipofected DS RRV particles in nonpermissive cells is caused by the DS component of the preparation and not to contaminating SS particles. This observation implies that uncoating of DS RRV particles in L and HEp2 cells can occur if the virus reaches the appropriate compartment and that the primary defect in nonpermissive cells is probably in the ability of the infecting virus to reach the cytoplasm after surface binding and internalization.

$D E A E$-dextran effects on RRV infection. Polycations have been shown to increase the infectivity of several viruses (20, 21 ) and enhance plaque formation by some strains of rotavirus $(22,23)$. Wishing to ensure that the effect of Lipofectin ${ }^{\mathrm{TM}}$ was not merely enhanced binding by some electrostatic mechanism, we examined the effects of DEAE dextran at a range of concentrations from $50-1,000 \mu \mathrm{g} / \mathrm{ml}$ during DS RRV viral

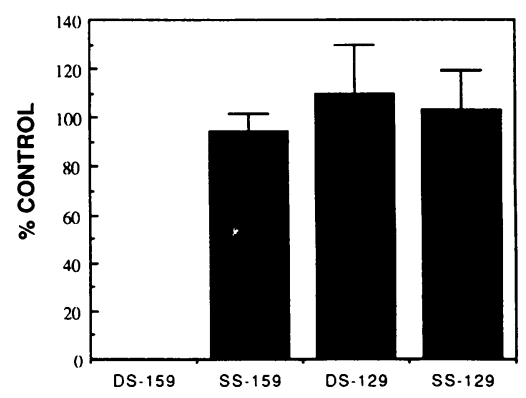

Figure 7. Effect of monoclonal antibodies on liposome-mediated transfections of SS and DS particles in Ma 104 cells. Particles were preincubated with the indicated $\mathrm{mAb}$ ascitic fluids at a 1:500 dilution for $30 \mathrm{~min}$ before transfection, as described in the text. Antigen-positive cells were detected by immunoperoxidase staining. Results are expressed as a percentage of control antigen positive cells observed with transfection of an equal quantity of untreated (no $\mathrm{mAb}$ ) SS or DS particles. ( $n=3$, SE indicated) 
adsorption and cell entry on all four cell lines. No significant effect was observed at any concentration on any of the cell lines. At the concentrations tested, DEAE dextran failed to mediate detectable cell infection by SS RRV particles. Thus, it appears unlikely that nonspecific electrostatic forces alone mediated the enhanced infectivity observed with the cationic liposomes.

Effect of trypsin activation on lipofection of $D S R R V$. As mentioned above, proteolytic activation of rotavirus by trypsin results in cleavage of $\mathrm{vp} 4$ and greatly enhanced efficiency of infection. It has been postulated that this cleavage promotes the penetration of the cell membrane by viral particles (10). Alternatively, trypsin activation might be a result of more efficient uncoating of the trypsin treated particles. To examine whether trypsin activation has any effect on lipofected DS particles, RRV was grown and purified without trypsin in the presence of aprotinin. The lack of cleavage of $\mathrm{vp} 4$ was verified by SDSPAGE before its use either with or without trypsin treatment to infect or lipofect $L$ cells. L cells were chosen for this experiment to minimize the amount of natural infection which might be occuring in the permissive lines. Fig. 8 depicts the results of such an experiment. Although trypsin did increase the infectivity of rotavirus $\sim 10$-fold over untreated virus in standard infections of $\mathrm{L}$ cells ( $O O$ vs $T O$, Fig. 8), in Lipofectin ${ }^{\mathrm{TM}}$-mediated infections the effect was much less ( $O L$ vs $T L$, Fig. 8).

\section{Discussion}

Cellular tropism of viruses in vivo is important in the pathogenesis of viral diseases. Understanding the basis for the restriction of viral growth in nonpermissive cells may offer insight into viral replicative strategies, as well as normal host cell functions. Such knowledge may be useful in devising strategies for the treatment and prevention of viral disease. In this paper, we have attempted to systematically examine each step in viral replication in permissive and relatively nonpermissive cells to understand at what stage replication is blocked. We used a technique of transfection of intact icosahedral viral and subviral particles by cationic liposomes to demonstrate that the mechanism of cellular tropism of rotavirus for certain cell lines probably occurs at the level of viral penetration of the plasma membrane, either directly from the cell surface or from endocytic vesicles.

We began our studies by identifying cell lines with a range of permissivity for RRV. We were unable to identify any ad-

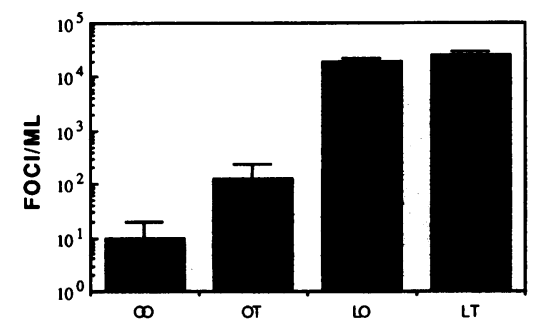

Figure 8. Effect of trypsin activation of DS particles on lipofection efficiency. RRV was grown in presence of aprotinin as described in the text, purified, and the DS fraction used to infect $\mathrm{L}$ cell monolayers after typsin activation $(5 \mu \mathrm{g} / \mathrm{ml})$ or mock ac-

tivation. Infections were performed in the presence or absence of $\mathrm{Li}$ pofectin $^{\mathrm{TM}}(10 \mu \mathrm{g} / \mathrm{ml}) . O O$, no Lipofectin ${ }^{\mathrm{TM}}$ and no trypsin; $O T$, no Lipofectin $^{\mathrm{TM}}$ and trypsin activated; $L O$, with Lipofectin ${ }^{\mathrm{TM}}$ and no trypsin; $L T$, with Lipofectin ${ }^{\mathrm{TM}}$ and trypsin activated. Results are the means of triplicate infections with SE indicated. herent mammalian cell line that was absolutely nonpermissive for RRV, so we chose several cell lines that are known to support the replication of other RNA viruses. L cells were selected because they are a poor substrate for rotavirus infection but an excellent substrate for other members of the Reoviridiae such as reoviruses. Likewise, HEp2 cells are epithelial cells that are highly permissive for picornaviruses but relatively resistant to rotavirus infection. HT29 human intestinal epithelial cells have been previously reported to be useful for the isolation of human rotaviruses (14).

Our initial studies showed a 100-300-fold difference in infectious progeny from permissive to the relatively nonpermissive cell lines (Fig. $1 a$ ). Further investigation by immunoperoxidase antigen detection (Fig. $1 b$ ) demonstrated that each cell line produced a similar number of viral progeny per infected cell (Table I) and that the differences in yield could be largely attributed to a much smaller portion of the $L$ and HEp2 cells producing RRV antigen. We concluded that viral assembly and release were not major determinants of the lower yields and that an early step in the replication cycle must account for the inefficient infection in L and HEp2 cells. Northern dot blot analysis of RRV RNA transcripts roughly paralleled the antigen production and suggested that efficiency of translation or posttranslational modification was not a major factor in the resistance of these cells to $R R V$ infection.

Because preliminary studies suggested that the defect observed in nonpermissive cells occurred early in the viral replication cycle, we initiated a series of experiments to examine viral binding and penetration. Radiolabeled RRV cell-binding studies and studies of infectious virus binding to cells failed to show significant differences between the permissive and nonpermissive cell lines (Figs. 3 and 4). Our studies did not address the possibility that the virus may bind to different receptors on the different cell lines used. In a previous report (2), we described the presence of high molecular weight suckling intestinal membrane glycoproteins, which when immobilized on nitrocellulose in a protein blot, specifically bound RRV. Because we have been unable to develop a similar assay for cultured cells (D. Bass, unpublished data), the relationship between the previously described putative intestinal receptor and the attachment of rotavirus to cultured cells in the present studies remains unclear.

When we analyzed the internalization of cell-bound virus by measuring escape of the radiolabeled inoculum from the cell surface by protease treatment of intact cells, we again found no significant differences between permissive and nonpermissive cell lines possibly because of endocytosis of large amounts of virus in nonproductive pathways in all cell lines. Of note, analysis of the fate of infectious virions that were removed from the cell surface (Fig. 4) revealed that internalized virus retained infectivity in L cells and HEp2 cells for a prolonged period. In contrast, the permissive cell lines that internalized an equal amount of radiolabeled virion contained very little infectious virus. These data suggested that the internalized virus in the nonpermissive cells was not efficiently uncoating to the singleshell transcriptionally active noninfectious form, perhaps because of sequestration of virions in endocytic vesicles and failure of the internalized virus to enter a low calcium environment, such as exists in the cell cytoplasm. It has been hypothesized that rotavirus uncoats when double-shelled particles are exposed to the extremely low cytoplasmic calcium concentrations during or after penetration of the plasma mem- 
brane. Ludert et al. demonstrated that calcium ionophores which increase cytosol calcium levels can block the intracellular uncoating of rotavirus (18). Thus in the nonpermissive cells infection could be blocked at either the membrane penetration step or because of an uncoating defect in the cytoplasm of nonpermissive cells.

To resolve this issue, we used a method of bypassing the penetration of membrane by using cationic liposomes to mediate delivery of viral particles directly into the cytoplasm. Initial experiments (Figs. 6, $a$ and $b$ ) showed that transfected single shell particles could mediate infection in all of the cell lines to a similar degree. This confirmed that the block to RRV replication lay in either penetration or uncoating, since all of the subsequent replicative events appeared equally efficient once the SS particle was transfected. To determine whether an uncoating block was important, we performed similar transfections with double-shelled RRV. Again, we observed comparable efficiency of infection in all of the cell lines, suggesting that uncoating of DS particles was not blocked in the nonpermissive cells, and that membrane penetration of virus into the cytosol appeared to be the rate-limiting step in the nonpermissive cells.

The mechanism by which rotavirus penetrates the plasma membrane to initiate infection in the cell cytoplasm is controversial. Some ultrastructural studies have suggested that endocytosis of rotavirus via coated pits is an important component of the process $(24,25)$ while others have reported observations of direct viral penetration of the cell membrane as the productive method of viral entry $(26,27)$. Studies using lysosomotrophic agents and inhibitors of endocytosis have reported little effect on rotavirus replication $(10,16,18,28)$. Trypsin activation is essential for efficient replication of rotavirus and it has been hypothesized that the cleavage of rotavirus outer capsid protein vp4 by trypsin facilitates membrane penetration (10). Consistent with this hypothesis, vp4 contains a hydrophobic amino acid domain which resembles alpha-virus fusion proteins (29). Furthermore the gene encoding vp4 has been genetically linked to a number of properties including growth in Mal04 (6) and HepG2 cells (7), and virulence (30). Vp4 forms a spikelike structure on the rotavirus particle (31) and may mediate binding to cells $(2,13)$. Kaljot et al. reported that trypsin-activated RRV but not unactivated RRV mediates the release of radioactive chromium from preloaded cells (10), suggesting that viral membrane penetration occurs at the cell surface. They also reported that release of chromium by activated virus was minimal in a rotavirus resistant cell line (bovine aortic endothelial cells) suggesting that these cells did not allow rotavirus penetration of the plasma membrane. Very recently, other workers have added support to the notion that trypsin cleavage of $\mathrm{vp} 4$ mediates plasma membrane penetration by showing that cleaved but not uncleaved rotavirus virions mediate the release of a fluorescent dye from artificial liposomes (32). Our data also lend support to this hypothesis in that trypsin treatment had little effect on the efficiency of rotavirus infection if the penetration step was mediated by lipofection (Fig. 8). While the present studies suggest that trypsin cleavage is important in membrane penetration, they do not identify the actual site of penetration which could be either from the cell surface and/or from some type of endocytic vesicle.

A block in replication in nonpermissive cells at the level of membrane penetration and/or uncoating has been demonstrated in several other viral systems including mumps virus
(33), foot-and-mouth disease virus ( 34 ), encephalomyocarditis virus (35), murine coronavirus (36), and adenovirus (37). In the case of encephalomyocarditis virus infection of rat cell lines the block was shown to be completely bypassed by transfection of viral RNA (35). Because we were able to transfect complete viral particles rather than nucleic acid, we were able to determine more precisely the nature of the inefficient rotavirus replication in nonpermissive cells. It remains unclear whether the observed low level of infection observed in the nonpermissive cells is caused by a general inefficiency of rotavirus penetration or whether a small subpopulation of the cells are fully permissive. We favor the former hypothesis because the observed rate of infection in L and HEp2 cells is directly proportional to the multiplicity of infection used.

Our study also suggests potential artifacts in the study of virus entry into cells. It is important that a distinction is made between entry, defined as virus escape from cell surface treatments such as proteases and antibodies that may occur via nonproductive endocytotic routes, and actual plasma membrane penetration into the cytoplasm, which is necessary for productive rotavirus infection.

Lipofectin ${ }^{\mathrm{TM}}$ consists of preformed cationic liposomes containing the synthetic lipid, DOTMA, which have been used for facilitation of transfection of nucleic acids. The mechanism by which Lipofectin ${ }^{\mathrm{TM}}$ mediates transfection is not entirely established, but it is believed that the liposomes adsorb nucleic acid to their surface rather than containing the DNA within a central cavity (38). We would postulate a similar mechanism for rotavirus particles, since duration or vigor of mixing of the Lipofectin $^{\text {TM }}$ with the particles had little effect on the efficiency of transfection.

Cationic liposomes and polyethylene glycol have been used to induce infection of nonpermissive cells by the enveloped viruses ecotropic murine leukemia virus and murine hepatitis virus respectively $(36,39)$. Our report differs from previous studies in that rotavirus has no outer membrane component and we were able to achieve a higher efficiency of infection in our system. Whether liposomes will prove useful as a general strategy to enhance infection with icosahedral viruses remains to be seen.

While our study does not address the actual mechanism of cell membrane penetration by rotaviruses, it does suggest the critical role of this step in the replicative cycle. The simplest model that could explain our results would be that susceptible cells such as Ma 104 and HT29 cells possess specific cell surface components which facilitate plasma membrane penetration by the virus. Resistant cells such as $L$ and HEp2 cells possess surface molecules that bind rotavirus but are unable to mediate penetration of the virus into the cytoplasmic compartment efficiently. The further evaluation of such a model will require the definite identification of a rotavirus cell surface receptor and/ or other factors linked to plasma membrane penetration.

\section{Acknowledgments}

This work was supported by Public Health Service Grant R22 AI21362, Digestive Disease Center Grant DK38707, and an American Gastroenterology Association Industry Scholar award to Dr. Bass.

\section{References}

1. Uhnoo, I., T. M. Riepenhoff, T. Dharakul, P. Chegas, J. E. Fisher, H. B. Greenberg, and P. L. Ogra. 1990. Extramucosal spread and development of hepa- 
titis in immunodeficient and normal mice infected with rhesus rotavirus. J. Virol. 64:361-368.

2. Bass, D. M., E. Mackow, and H. B. Greenberg. 1991. Identification and partial characterization of rhesus rotavirus binding glycoprotein on murine enterocytes. Virology. 183:602-610.

3. Riepenhoff, T. M., P. C. Lee, P. J. Carmody, H. J. Barrett, and P. L. Ogra. 1982. Age-dependent rotavirus-enterocyte interactions. Proc. Soc. Exp. Biol. Med. 170:146-154.

4. Vonderfecht, S. L., R. L. Miskuff, S. B. Wee, S. Sato, R. R. Tidwell, J. D. Geratz, and R. H. Yolken. 1988. Protease inhibitors suppress the in vitro and in vivo replication of rotavirus. J. Clin. Invest. 82:2011-2016.

5. Bass, D. M., M. Baylor, R. Broome, and H. B. Greenberg. 1992. Molecular basis of age-dependent gastric inactivation of rhesus rotavirus in the mouse. $J$. Clin. Invest. 89:1741-1745.

6. Greenberg, H. B., J. Flores, A. R. Kalica, R. G. Wyatt, and R. Jones. 1983. Gene coding assignments for growth restriction, neutralization and subgroup specificities of the W and DS-1 strains of human rotavirus. J. Gen. Virol. 64:313320.

7. Ramig, R. F., and K. L. Galle. 1990. Rotavirus genome segment 4 determines viral replication phenotype in cultured liver cells (HepG2). J. Virol. 64:1044-1049.

8. Kalica, A. R., J. Flores, and H. B. Greenberg. 1983. Identification of the rotaviral gene that codes for hemagglutination and protease-enhanced plaque formation. Virology. 125:194-205.

9. Shaw, R. D., P. T. Vo, P. A. Offit, B. S. Coulson, and H. B. Greenberg 1986. Antigenic mapping of the surface proteins of rhesus rotavirus. Virology. 155:434-451

10. Kaljot, K. T., R. D. Shaw, D. H. Rubin, and H. B. Greenberg. 1988. Infectious rotavirus enters cells by direct cell membrane penetration, not by endocytosis. J. Virol. 62:1136-1144.

11. Paeratakul, U., P. R. De Stasio, and M. W. Taylor. 1988. A fast and sensitive method for detecting specific viral RNA in mammalian cells. J. Virol. 62:1132-1135.

12. Cohen, J., F. Lefevre, M. K. Estes, and M. Bremont. 1984. Cloning of bovine rotavirus (RF strain): nucleotide sequence of the gene coding for the major capsid protein. Virology. 138:178-182.

13. Ruggeri, F. M., and H. A. Greenberg. 1991. Antibodies to the trypsin cleavage peptide $\mathrm{vp} 8^{*}$ neutralize rotavirus by inhibiting binding of virions to target cells in culture. $J$. Virol. 65:2211-2219.

14. Superti, F., A. Tinari, L. Baldassari, and G. Donelli. 1991. HT-29 cells: a new substrate for rotavirus growth. Arch. Virol. 116:213-218.

15. Clark, S. M., J. R. Roth, M. L. Clark, B. B. Barnett, and R. S. Spendlove. 1981. Trypsin enhancement of rotavirus infectivity: mechanism of enhancement. J. Virol. 816-822.

16. Fukuhara, N., O. Yoshie, S. Kitaoka, and T. Konno. 1988. Role of VP3 in human rotavirus internalization after target cell attachment via VP7. J. Virol. 62:2209-2218.

17. Suzuki, H., S. Kitaoka, T. Konno, T. Sato, and N. Ishida. 1985. Two modes of human rotavirus entry into MA 104 cells. Arch. Virol. 85:25-34.

18. Ludert, J. E., F. Michelangeli, F. Gil, F. Liprandi, and J. Esparza. 1987. Penetration and uncoating of rotaviruses in cultured cells. Intervirology. 27:95101.

19. Cohen, J., J. Laporte, A. Charpilienne, and R. Scherrer. 1979. Activation of rotavirus RNA polymerase by calcium chelation. Arch. Virol. 177-182.
20. Kooi, C., L. Mizzen, C. Alderson, M. Daya, and R. Anderson. 1988. Early events of importance in determining host cell permissiveness to mouse hepatitis virus infection. J. Gen. Virol. 69:1125-1135.

21. Bailey, C. A., D. K. Miller, and J. Lenard. 1984. Effects of DEAE-dextran on infection and hemolysis by VSV. Evidence that nonspecific electrostatic interactions mediate effective binding of VSV to cells. Virology. 133:111-118.

22. Matsuno, S., S. Inouye, and R. Kono. 1977. Plaque assay of neonatal calf diarrhea virus and the neutralizing antibody in human sera. J. Clin. Microbiol. $5: 1-4$.

23. Hirano, N., F. Sato, K. Ono, T. Murakami, and M. Matumoto. 1987. A sensitive plaque assay for bovine rotavirus in cultures of the bovine cell line GBK Vet. Microbiol. 13:383-387.

24. Quan, C. M. and F. W. Doane. 1983. Ultrastructural evidence for the cellular uptake of rotavirus by endocytosis. Intervirology. 20:223-231.

25. Ludert, J. E., F. Michelangeli, F. Gil, F. Liprandi, and J. Esparza. 1987. Penetration and uncoating of rotaviruses in cultured cells. Intervirology. 27:95101.

26. Suzuki, H., S. Kitaoka, T. Konno, T. Sato, and N. Ishida. 1985. Two modes of human rotavirus entry into MA 104 cells. Arch. Virol. 85:25-34.

27. Suzuki, H., S. Kitaoka, T. Sato, T. Konno, Y. Iwasaki, Y. Numazaki, and N. Ishida. 1986. Further investigation on the mode of entry of human rotavirus into cells. Arch. Virol. 91:135-144.

28. Keljo, D. J., M. Kuhn, and A. Smith. 1988. Acidification of endosomes is not important for the entry of rotavirus into the cell. J. Pediatr. Gastroenterol. Nutr. 7:257-263.

29. Mackow, E. R., R. D. Shaw, S. M. Matsui, P. T. Vo, M. N. Dang, and H. B. Greenberg. 1988. The rhesus rotavirus gene encoding protein VP3: location of amino acids involved in homologous and heterologous rotavirus neutralization and identification of a putative fusion region. Proc. Natl. Acad. Sci. USA. 85:645-649.

30. Offit, P. A., G. Blavat, H. B. Greenberg, and H. F. Clark. 1986. Molecular basis of rotavirus virulence: role of gene segment 4. J. Virol. 57:46-49.

31. Prasad, B. V., J. W. Burns, E. Marietta, M. K. Estes, and W. Chiu. 1990 Localization of VP4 neutralization sites in rotavirus by three-dimensional cryoelectron microscopy. Nature (Lond.). 343:476-9.

32. Nandi, P., A. Charpilienne, and J. Cohen. 1992. Interaction of rotavirus particles with liposomes. J. Virol. 66:3363-3367.

33. Yamada, A., M. Tsurudome, M. Hishiyama, and Y. Ito. 1984. Abortive infection of mumps virus in murine cell lines. J. Gen. Virol. 184:973-980.

34. Clarke, J. B., and R. E. Spier. 1983. An investigation into causes of resistance of a cloned line of BHK cells to a strain of foot-and-mouth disease virus. Vet. Microbiol. 8:259-270.

35. Donta, S. T., J. D. Shanley, and B. P. Damiano. 1986. Encephalomyocarditis virus can bind to and transfect non-permissive cells. Arch. Virol. 88:301307.

36. Van Dinter, S., and W. F. Flintoff. 1987. Rat glial C6 cells are defective in murine coronavirus internalization. J. Gen. Virol. 68:1677-1685.

37. Horvath, J., and J. M. Weber. 1988. Nonpermissivity of human peripheral blood lymphocytes to adenovirus type 2 infection. J. Virol. 62:341-345.

38. Felgner, P. L. and G. M. Ringold. 1989. Cationic liposome-mediated transfection. Nature (Lond.). 337:387-388.

39. Innes, C. L., P. B. Smith, R. Langenbach, K. R. Tindall, and L. R. Boone 1990. Cationic liposomes (Lipofectin) mediate retroviral infection in the absence of specific receptors. J. Virol. 64:957-961. 